\title{
Strengthening Human Resources for Health to Achieve Epidemic Control: A Case Study of Democratic Republic of the Congo
}

Paul W. Thurman ( $\square$ paul.thurman@columbia.edu )

https://orcid.org/0000-0003-3053-6255

\section{Susan Michaels-Strasser}

Columbia University Mailman School of Public Health

Luc Ferdinand Kamanga Lukusa

Columbia University Mailman School of Public Health

John Ngulefac

Health Resources and Services Administration

Beatrice Lukeni

Columbia University Mailman School of Public Health

Serge Matumaini

Columbia University Mailman School of Public Health

Noe Assukulu

Columbia University Mailman School of Public Health

Lauren Parmley

Columbia University Mailman School of Public Health

Sungani Zidana-Ndovi

Columbia University Mailman School of Public Health

Faustin Malele

Columbia University Mailman School of Public Health

\section{Case study}

Keywords: Human resources for health planning, HIV/AIDS epidemic control, $\mathrm{HRH}$ intervention prioritization, optimal utilization of health workers, health worker capacity strengthening, PEPFAR 95-9595 MER target attainment

Posted Date: July 17th, 2020

DOl: https://doi.org/10.21203/rs.3.rs-41976/v1 
License: (c) (i) This work is licensed under a Creative Commons Attribution 4.0 International License. Read Full License 


\section{Abstract}

Background The United States President's Emergency Plan for AIDS Relief (PEPFAR) in Democratic Republic of the Congo (DRC) continues to fund a robust portfolio of programs aimed at achieving epidemic control in three provinces where 30 percent of people living with HIV/AIDS in the country reside. Challenges around human resources for health $(\mathrm{HRH})$, including inadequate staffing and limited capacity, impede the delivery of quality HIV/AIDS services in DRC.

Methods In partnership with the United States Health Resources and Services Administration (HRSA), PEPFAR, and DRC Ministry of Health (MoH), ICAP at Columbia University worked with 16 PEPFARidentified high-priority health facilities (HFs) in DRC and developed $\mathrm{HRH}$-specific interventions to address challenges in achieving 95-95-95 targets. These potential interventions were then prioritized for implementation using a collaborative, criteria-driven approach considering factors such as feasibility, viability, and time-to-impact. Through interviews at all $16 \mathrm{HFs}$, the joint teams developed an intervention framework, determined short-term priorities, and prepared to implement short-term HRH improvements to reach 95-95-95 targets across all HFs. Interviews used an adapted version of the PEPFAR HRH Rapid Assessment tool to capture key HRH information including staffing levels by type of clinical or administrative position, key barriers to achieving 95-95-95 targets, and perceptions of needed $\mathrm{HRH}$ specific improvements.

Results Site-level interviews occurred in April 2019, and the in-country team created a list of possible interventions across six domains: staffing, training, workplace environment, medical supplies and equipment, and monitoring and evaluation. Thirty-five interventions were hypothesized and prioritized into short, medium, and long-term priorities using a framework focused on desirability, feasibility, viability, and time-to-impact. Some interventions were applicable to all HFs while others applied only to selected $\mathrm{HFs}$, the national $\mathrm{MoH}$, or to implementation partners. Twelve interventions were selected as highest priority, and budget allocations and task planning were developed for each of the high-priority interventions. These high-priority interventions were then launched for implementation and evaluation within six months.

Conclusions The supply and quality of $\mathrm{HRH}$ are critical to achieving epidemic control. This assessment delineated necessary interventions to address site-specific HRH barriers, $\mathrm{HRH}$ interventions focused on ensuring adequate staffing, optimal utilization of health workers, and strengthening health workers' capacity to provide quality HIV/AIDS services to achieve epidemic control. Downstream tracking and reporting of key PEPFAR metrics, including key Monitoring, Evaluation, and Reporting (MER) indicators, will allow intervention teams to conduct program evaluations of key interventions and their impacts on PEPFAR targets.

\section{Background}




\section{Democratic Republic of the Congo (DRC) Context and Challenges}

Democratic Republic of the Congo (DRC) gained independence in 1997 and has received United States President's Emergency Plan for AIDS Relief (PEPFAR) funding since PEPFAR's inception in 2003 [1, 2]. DRC is a country of over 2 million square kilometers that borders seven sub-Saharan African countries. Decades of violent conflict and instability have taken a devastating toll on the country's economy, human resources, and infrastructure, and, coupled with a largely rural population, present several challenges to providing healthcare [3].

DRC has a generalized HIV epidemic with an estimated prevalence of 1.2 percent among adults aged 15 to 49 . HIV infection is more prevalent in urban settings ( 1.6 percent) than rural areas ( 0.9 percent). An estimated 404,894 people are living with HIV/AIDS, and around 10,535 people die from AIDS related conditions each year [4]. The HIV/AIDS epidemic has drastically impacted two of DRC's most populous provinces, with general population prevalence estimates of 1.6 percent in Kinshasa and 2.6 percent in Haut Katanga [5].

ICAP at Columbia University initiated comprehensive support of HIV/AIDS care and treatment (C\&T) services in DRC in 2010. Since then, ICAP has worked hand-in-hand with the Programme National de Lutte contre le SIDA (PNLS) to expand the availability, quality, and uptake of adult and pediatric HIV/AIDS C\&T in DRC with an emphasis on expanding prevention of mother-to-child transmission (PMTCT) activities, development of a laboratory network for disease monitoring, integration of HIV/AIDS and tuberculosis (TB) services, improvement of infrastructure, prevention among key populations (KP) in Kinshasa and Haut Katanga provinces, and epidemic control. In partnership with DRC Ministry of Health $(\mathrm{MoH})$ and donors, ICAP has rapidly expanded its support for PNLS activities from just 10 sites in 2010 to 240 public and private hospitals, health centers, and TB clinics in Kinshasa and Haut Katanga provinces as of September 2014. ICAP currently supports 199 sites.

\section{Human Resources for Health (HRH): Priorities and Challenges}

Strengthening $\mathrm{HRH}$-in terms of quantity, quality, and distribution-has been a priority focus of many global public health and epidemic control efforts, especially in African nations struggling to control outbreaks of malaria, TB, HIV/AIDS, Ebola, and other infectious diseases [6, 7]. In DRC, numerous training and policy measures have aimed to address gaps in managing a disparate and diverse healthcare workforce, including nurses, community health workers (CHWs), and midwives, across a geographically and culturally diverse country [8,9]. Since healthcare (and especially HIV/AIDS treatment) is often centralized at major health facilities (HFs) in large cities, decentralized efforts to reach DRC's largely rural population (often with higher infection rates) are constrained by lack of trained health care workers able to provide the full breadth of HIV/AIDS services. $[10,11]$. Further, the abilities of these community staff and HFs to influence local healthcare policies and procedures are constrained by distance, staff burnout 
and a sense that rural health services may not "matter" as much $[12,13]$. These challenges can lead to staff feeling underappreciated, burned out, or unclear of job responsibilities and expectations $[14,15]$. Thus, for ICAP and its partners and funders, implementing new HIV/AIDS-specific interventions can sound terrific in principle but can be quite difficult in practice.

In response to the urgent need to scale-up access to antiretroviral therapy (ART), in close collaboration with the Ministry of Health $(\mathrm{MoH})$ and Nursing Council and Associations, ICAP successfully implemented a PEPFAR-funded program administered by the United States Department of Health Resources and Services Administration (HRSA), the Global Nursing Capacity Building Project (GNCBP), across 10 countries including DRC between 2011 and 2018. Working closely together, HRSA and ICAP partnered with in-country nursing leaders and ministries of health and education $(\mathrm{MoH} / \mathrm{MoE})$ to strengthen the nursing and midwifery workforce. This project addressed critical gaps in nursing education and training focusing on seven building blocks of health workforce strengthening: infrastructure improvement, curricula revision, clinical skills development, production, in-service training and faculty development and building partnerships with $\mathrm{MoH}$ and MoE stakeholders for policy and regulation with the overarching goal of enhancing the quality and quantity of the nursing and midwifery workforce. Therefore, ICAP and HRSA have a demonstrated track record of collaborative success in HRH improvements in DRC working at both the pre- and in-service levels.

\section{Epidemic Control and PEPFAR}

In support of the Joint United Nations Programme on HIV/AIDS (UNAIDS) 95-95-95, PEPFAR-supported programs place emphasis on those activities which are proximal to reaching these targets and rapid treatment initiation and clinic and community efforts which focus on retention in care and viral suppression. Therefore, this paper discusses efforts to strengthen HRH at 16 PEPFAR-identified "highpriority" HFs in the Haut Katanga region towards achievement of 95-95-95 goals. These HFs were prioritized based on the volume of patients on ART and regional HIV/AIDS prevalence and, as such, appears to be a good target collaboration between ICAP and HRSA/PEPFAR collaborations. The HIV/AIDS prevalence in Haut Katanga is 2.5 percent compared to 1.2 percent in the capital, Kinshasa and is challenged by a largely rural population and large land mass borders with both Zambia (HIV/AIDS prevalence of 11.4 percent among adults aged 15-49) and Malawi (10.0 percent among adults aged 1549) $[5,16,17]$.

\section{Resilient and Responsive Health Systems (RRHS) Projects}

With PEPFAR funding through HRSA, in 2017, ICAP was awarded the RRHS project to continue strengthening $\mathrm{HRH}$ in DRC. Using the World Health Organization (WHO) conceptual framework for HRH development, the first two years of the RRHS project built on extensive HRH capacity building and infrastructure improvements for student nurses and midwives through HRSA funding the Nursing Education Partnership Initiative (NEPI) as a follow-on to the previously funded GNCBP [18]. Since nurses are the largest HRH component in DRC, this NEPI-focused work centered on nurses' readiness for clinical practice through curricula reform and development of innovative pedagogy including use of skills labs 
and simulation-based training. Over the past two years, HRSA and ICAP have leveraged these pre-service strengthening efforts to shift focus to in-service capacity building and addressing wider HRH limitations affecting epidemic control. Improving clinic personnel collaboration, implementing healthcare worker and management training in HIV/AIDS and other leadership skills, upgrading infrastructure for HRH effectiveness, and improving morale and job stability via rural rotations of nursing school graduates are all examples of RRHS activities that focus on HRH in DRC.

The value-added of such HRH interventions extends beyond C\&T programs and thus may support faster achievement of PEPFAR 95-95-95 goals. These faster outcomes are well-known from prior research as are the pitfalls if such HRH needs are not addressed [19-21]. Thus, this paper outlines ICAP's method to develop targeted, prioritized, and sequenced interventions-at the HF, MoH, and ICAP levels-to improve $\mathrm{HRH}$ effectiveness. With improved HRH effectiveness, ICAP and HRSA hope to demonstrate faster and more efficient achievement of 95-95-95 goals than would have been possible with only C\&T interventions, alone [22]. Further, such HRH improvements can inform country- and regional public health policy to more accurately and quickly identify health workforce needs moving forward [8].

\section{Methods}

A needs-driven process was used to determine site-level HRH needs across the PEPFAR-identified 16 highpriority HRs and to develop efficient action plans to address these needs within 12-18 months. This protocol was submitted to the Columbia University Irving Medical Center Institutional Review Board (IRB) and received a "non-research" determination. A series of 13 steps were completed between October 2018 and June 2019 after which HRH interventions were launched in early July 2019:

1. The PEPFAR Rapid Site-Level Health Workforce Assessment (hereafter, "HRH assessment") was modified and translated into French $[23,24]$. The modified assessment collected no personal information-such as names of HF staff or management-and focused on challenges that HRH faced, not "time and motion" information regarding daily staffing, vacations, etc. Exhibits 1A, 1B, and $1 \mathrm{C}$ provide excerpts of the original English $\mathrm{HRH}$ assessment tool, the French translation of the same, and a section of the (French-language) Microsoft Excel data collection tool, respectively

2. Ten high-priority sites were selected for survey piloting. Local ICAP staff were trained on the survey and then scheduled initial interviews with HF leaders and staff. All participants were advised that no personal information would be logged and that all responses would be anonymous. Any participant could opt-out of the interview-in whole or in part-at any time

3. Once the ten pilot site interviews were completed, the ICAP team reviewed initial findings and suggestions for how to improve/streamline the survey questions and interviews

4. All 16 sites were then interviewed-including re-interviews of the ten pilot sites-with the modified $\mathrm{HRH}$ assessment and presentation of current Monitoring, Evaluation, and Reporting (MER) statistics for each site [25]. Exhibits 2A, 2B, and 2C show MER snapshot statistics for one of the high-priority HFs in DRC. (Each sub-exhibit is one of the three "95-95-95" MER sets.) Any target coded "green" was 
considered sufficiently met. Yellow and red colors indicate improvements needed or lagging performance relative to PEPFAR expectations

5. The team reviewed all data and prepared summaries of key needs from HF interviews. This helped the team establish top HRH challenges and needs for each site

6. The team then assessed volume data-of patients, clinical staff, etc.-to determine what training gaps existed and where excessive patient loads were across the HFs. This allowed the team to determine if site-level HRH needs were related to patient volumes, to numbers of HIV/AIDS trained staff, or to other factors beyond the C\&T domain

7. From this work several hypotheses for interventions that could address key HRH needs/challenges were developed. These were discussed with the local C\&T teams to determine if any of findings were misaligned with C\&T goals and improvements already scheduled

8. Interventions were then prioritized using a prioritization framework with criteria such as desirability, feasibility, and time-to-impact (see detailed discussion below). Priorities were shared with key HF and HRSA leaders for review and comment. Prioritization of interventions was informed by frameworks used in similar contexts in other infectious disease treatment areas and African nations [26, 27].

9. Interventions were then determined to be of short-term focus (within the first six months, mediumterm (to be done in months 7-12), or long-term (to be completed in months 13-18, or by December 2020)

10. Once agreed, $\mathrm{HRH}$ interventions were slotted as being the responsibility of specific sites, all sites, the $\mathrm{MoH}$, or ICAP

11. Short-term HRH interventions-including establishing a July 2019 baseline of 95-95-95 MER statistics for future benchmarking-were then launched

12. Concomitant with short-term intervention launch, the team developed a tracking tool, comprising both C\&T and HRH intervention efforts, that aligned efforts with all 95-95-95 targets

13. Since mid-2019, monthly reporting of MER data-and of key C\&T and HRH intervention progresshas been provided to all in-country leadership and HRSA. Bi-weekly conference calls with in-country leaders, ICAP staff, and HRSA leadership are held to assess line-item progress and exception handling

The collaborative process used focused on building trust with sites and empowering HF leaders to express needs for HRH improvement in ways the team could report back to funders (HRSA) and MoH. Building relationships with HF leaders was critical to success of any planned intervention especially to upscaling staffing, upskilling health workers, and improving workplace conditions and culture. Trustbuilding is especially important when providing financial incentives for performance is impossible and quickly adding qualified staff is unlikely [28-30].

\section{Results}

All 16 high-priority HFs responded in detail to the HRH assessment and participated fully in on-site interviews. Results from the team's initial data collections indicated that, contrary to initial conjecture, gross staffing level challenges were generally not an issue at the HFs. Exhibit 3 shows the staffing across 
all 16 sites by type of staff (e.g., doctor, nurse, midwife) and those who were trained in HIV/AIDS care and treatment. Full-time vs. part-time staffing is also noted. Total staffing numbers include non-HIV/AIDStrained staff, but for the most part, HFs in aggregate were well-staffed for HIV/AIDS care.

An evaluation of HIV/AIDS caseload was then performed to determine if appropriate levels of HIV/AIDStrained staff were in place at each HF. Exhibit 4 shows the HIV/AIDS case load (end of March 2019) for each site with the average load shown as a horizontal line. While midwives are needed across the board, only four sites (shown in red) required more HIV/AIDS-qualified health staffing (nurses), and only one site (shown in green) required more doctors and nurses. Thus only four of the $16 \mathrm{HFs}$ required additional trained staff-not necessarily hired staff-in HIV/AIDS care and treatment. Only one site required increased (clinical) staffing.

From these analyses, the team concluded that having enough staff was not a key $\mathrm{HRH}$ issue and that, instead, other needs likely deserved more attention (aside from the clear HRH resource needs in the five sites mentioned in Exhibit 4). After compiling the qualitative results from the HF interviews, Exhibit 5 offers some interesting insights into HRH challenges in the HFs:

- While few barriers seem to be compensation-related, more HIV/AIDS clinical staff and training (clinical and non-clinical) are needed. Low morale seems tied to lack of job descriptions, evaluations, and career pathing

- Improvements in morale could lead to better recruiting and/or positive selection for employment at HFs, which could help "up-scale" these HFs [31, 32]

- Community activities are not well-organized due to lack of peer educators and community health worker training and oversight. Inconsistent oversight of and non-standard documentation from these workers is not only in the HIV/AIDS space but also in TB and malaria domains

- Work environments require improvement-spaces to collaborate, rest, etc.-and workers need more protective clothing and communities of interest to share / learn best practices

- Improved ART distribution would help as would access to more advanced therapies. Budgeting support will also help some individual HFs [33]

After a careful analysis of $\mathrm{HF}$ interview data, the team reviewed literature on $\mathrm{HRH}$ intervention research and determined that the barriers, above, could be addressed with interventions across the following six domains [34]:

- Staffing: Increasing clinical hiring (doctors, nurses, midwives) and peer educators, and optimizing time allocated to HIV/AIDS patients by these clinical resources

- Training: Training CHWs in HIV/AIDS-specific care protocols, improving job descriptions and expectations to enhance morale, and enhancing collaboration and management skills

- Workplace Environment: Providing opportunities for, renovating sites, implementing worker protections, and rewarding community involvement and site leadership 
- Community Outreach: Working to bring more patients into the clinic, increasing screenings, and creating communities of interest to share best practices (sites and communities)

- Medical Supplies and Equipment: Providing access to advanced drugs and equipment, improving supply chains for ARTs, and helping sites better budget and report 95-95-95 progress

- Monitoring and Evaluation: Standardizing monthly reviews of 95-95-95 progress and collecting ongoing feedback on barriers to HRH effectiveness [35]

Determining responsible stakeholders for these interventions is also critical [36]. The team determined that four key stakeholders would be assigned responsibilities for any interventions across the six domains, above. Interventions would be implemented by all sites, selected sites, the $\mathrm{MoH}$, and/or by ICAP. Exhibits $6 \mathrm{~A}$ and 6B show the "universe" of proposed interventions by key stakeholder/responsible party. For example, in the "Training" domain, "All Sites" will need to provide more HIV/AIDS-specific training while only one "Selected Site" will need to hire dedicated HIV/AIDS staff members. The MoH would need to approve budget for such training and personnel, and ICAP would need to provide the proper job description(s) and training plan for new hires or training programs for existing $\mathrm{HRH}$.

However, not all of these 35 interventions are of the same importance/urgency nor could all be launched simultaneously (assuming resources were even available to do so). Thus, the team constructed a set of prioritization criteria in order to stage and sequence these interventions in a more manageable way [37]:

- Desirability: Do HFs, the MoH, ICAP really want to pursue this intervention (based on past experience, etc.)?

- Feasibility: If the intervention is desirable, do HFs, the MoH, ICAP, etc. have the (non-financial) resources, skills, and tools ready to kick-off the intervention?

- Viability: If stakeholders have the skills and time, do they have the funding, or can they quickly get the needed it to fund the intervention?

- Sustainability: Once implemented, will the HRH interventions "stick;" i.e., will the improvements stand the test of time or be lost as just one-time changes?

- Time to Impact: Finally, if stakeholders implement an intervention, have skills to do it, and can be funded, will they see a return on their efforts based on meaningful and timely impacts?

After working with HFs, HRSA leadership, ICAP, and $\mathrm{MoH}$, the team prioritized the interventions, and Exhibit 7 shows the results of this collaboration. Efforts were prioritized in terms of what needed to be implemented in the short-term (within the first six months), over the medium-term (within the following six months), and over the long-term (within the final six months of the total intervention time frame of 18 months). Exhibit 8 shows only the short-term interventions noting that two of them had already been started by C\&T teams.

Based on successes of similar HRH interventions in other sub-Saharan contexts, the team expects to see several improvements [35]. These are aligned with the major HRH domains mentioned previously: 
- Staffing: Increase numbers of patients tested/tested positive for HIV/AIDS and those treated if positive (including pregnant women) as more qualified health workers, midwives, and peer educators are put into the $\mathrm{HRH}$ workforce

- Training: Install more staff able to help HIV/AIDS patients, improve satisfaction, and enhance clinic leadership. These should create more awareness for clinics (or "pull") for new and existing cases

- Workplace Environment: Improve workplace environments that lead to more team-based care, best practice implementations, and improved morale for staff

- Community Outreach: Create wider networks in the community and build trust with clinics. This will increase treated cases, follow-ups, lower viral loads, etc.

- Medical Supplies and Equipment: Deploy therapies to patients more quickly and give workers more security via enhanced protective equipment

- Monitoring and Evaluation: Place emphasis on achievement of 95-95-95 goals with standard reporting across clinics (and with $\mathrm{MoH}$ ) to quickly implement best practices

To track these expected improvements, the team developed a comprehensive reporting system, using Microsoft Excel, that incorporates both monthly MER data, to track C\&T improvements, and the HF interventions noted earlier. In this way, all stakeholders-HF leaders, C\&T teams, ICAP staff, MoH, and HRSA-are able to view monthly progress updates, note where efforts are not meeting desired goals, and see corrective actions being taken to address shortcomings. Exhibits 9A (MER statistics) and 9B (sitelevel HRH interventions) provide snapshots as of November 2019 for these DRC HRH efforts.

The team hopes to use these data to perform program evaluations to determine intervention- and program-level effectiveness and to show how these efforts have led to better outcomes, as measured by MER reporting, than C\&T interventions, alone, may have created.

\section{Discussion}

The tools developed-and preliminary results achieved in HRH effectiveness-would not have been possible without direct, site-level data collection and interviews from HF leaders (both clinical and nonclinical). By assuring respondents confidentiality of collected inputs, the team was able to obtain honest feedback about key HRH challenges and needs. The PEPFAR HRH rapid assessment tool proved useful in collecting these data quickly. Piloting the process first, and then revising the data collection tool also proved valuable since the team could incorporate learnings from early site interviews to make the entire data collection more effective.

Sustained contact with stakeholders throughout the processes of setting MER baselines and HRH staffing, hypothesizing possible interventions, prioritizing interventions, and launching and tracking implementations allowed the team to keep stakeholders aligned and focused on important endpoints throughout this study. Stakeholders agreed to the major domains of HRH needs, prioritization criteria, and the staged implementation plans. Since launch, stakeholders have appreciated the value of monthly MER 
reporting aligned with updates that show successes and challenges with corrective actions planned to mitigate poor performance (by month by HF).

Keeping focus on various stakeholder needs, too, has proven to be valuable as $\mathrm{MoH}$ priorities may not always align with HF needs (or those of funders, necessarily). Thus, with open communication and through monthly reviews and planning, these potential "de-railers" to HRH success can be made evident, planned for, and accommodated by multiple parties working collaboratively. This, in turn, has built trust across several constituents, all of whom have a vested interest in HIV/AIDS epidemic control through improved HRH staffing, training, and collaboration. Prioritization and sequencing of $\mathrm{HRH}$ interventions was key to successful implementation.

But without tracking-of both intervention progress and the direct results of interventions through MER results-any program will likely produce suboptimal or unmeasurable results. Thus, the close coordination of C\&T and HRH teams was a critical element of success, and this coordination has resulted in greater trust and improved knowledge-sharing across these teams.

The team looks forward to reporting more results from its evaluations of these $\mathrm{HRH}$ interventions-and their correlations to changes in MER improvements-in the coming months. Also as the team expands this study to non-PEPFAR funded areas/countries, it hopes to see similar results in other contexts (and, perhaps, beyond just HIV/AIDS-dominated areas).

\section{Limitations}

Some limitations to this approach are noteworthy. First, only PEPFAR high-priority HFs were interviewed for these targeted HRH interventions. Although the team believes these interventions are relevant to other $\mathrm{HFs}$, the HRH assessment did not compare priority to non-priority sites and has not identified possible divergent $\mathrm{HRH}$ needs.

Second, given different country contexts in which these interventions may apply, results may vary based on $\mathrm{MoH}$ adoption, in-country and external funding, and in-country HRH staffing and skills. The team hopes to pilot this study elsewhere to see if other HFs identify similar HRH needs and can realize similar gains from such interventions. National and local government priorities, which can change based on varying needs and "shocks," will always be exogenous factors that could affect any intervention efficacy.

\section{Conclusions}

While the correlation between number of health workers and care outcomes is well documented [22, 38], less evidence exists regarding $\mathrm{HRH}$ interventions that go beyond the staffing numbers. Here, the team determined that some HRH needs and challenges, for 16 high-priority PEPFAR HFs in DRC, were actually not related to staffing levels but to other factors including training, workplace environment, morale, and a need to feel/be connected to other HFs and to best practices. 
Staffing levels did not appear to be a significant HRH challenge-except for certain needs in certain sites. Getting staff properly trained, connected to each other, and aligned with formal job descriptions could yield significant benefits as measured by MER target achievement and staff morale, for example. Keeping staff trained, on task, and connected appear to be significant drivers of positive culture and therefore help clinical and non-clinical staff work better, together, to improve testing, treatment, and follow-up for those with HIV/AIDS as has been noted elsewhere.

By personally interviewing site leadership staff, developing interventions across key HRH domains, and prioritizing these with stakeholders, the team was able to quickly launch high-impact interventions that continue to bear fruit for high-priority HFs. However, ongoing tracking of both key 95-95-95 MER data and site-level HRH intervention progress is critical to correlate HRH interventions to epidemic control. The team hopes to pilot similar efforts at non-PEPFAR sites, both within DRC and in other countries, and report successes that could not have been achieved with C\&T initiatives, alone [39].

Success of these HRH interventions, then, can be linked to national and regional health policies, and HRH effectiveness can be more of an enabler than a constraint of epidemic control [40]. This can only enhance HRH efficacy and intervention sustainability [41].

\section{Abbreviations}

ART: Anti-Retroviral Therapy(ies)

C\&T: Care and Treatment

CDC: (United States) Centers for Disease Control and Prevention

CHW: Community Health Worker

DRC: Democratic Republic of the Congo

GNCBP: Global Nursing Capacity Building Program

HF: Health Facility

HIV/AIDS: Human Immunodeficiency Virus/Acquired Immune Deficiency Syndrome

$\mathrm{HRH}$ : Human Resources for Health

HRSA: (United States) Health Resources and Services Administration

IRB: Institutional Review Board

KP: Key Populations

MER: Monitoring, Evaluation, and Reporting 
MoE: Ministry of Education

MoH: Ministry of Health

NEPI: Nursing Education Partnership Initiative

PEPFAR: (United States) President's Emergency Plan for AIDS Relief

PMTCT: Prevention of Mother-To-Child Transmission

PNLS: Programme National de Lutte Contre le VIH/SIDA, DRC's national AIDS program

RRHS: Resilient and Responsive Health Systems

TB: Tuberculosis

UNAIDS: Joint United Nations Programme on HIV/AIDS

WHO: World Health Organization

\section{Declarations}

\section{Acknowledgements}

The authors wish to thank all study respondents, MoH, PNLS, HRSA, and ICAP leadership and staff for their time and ongoing commitment to HIV/AIDS epidemic control in DRC. The authors also thank Stella M. Lodewick, Glen Ridge High School, Glen Ridge, New Jersey, USA, for her assistance with background research on intervention policy and implementation and for her help organizing our bibliography and citations.

\section{Authors' Contributions}

PT developed the overall research methodology including interview guides, prioritization frameworks, and intervention plans. SM-S provided background on the PEPFAR, DRC, and HRH contexts and developed a reporting framework for site-level interventions. JN and LFKL contributed to the in-country implementation funding and prioritization. BL and SM provided in-country technical guidance and RRHS leadership (including site-level collaborations). NA and LP provided all strategic information reporting and tracking including MER indicators and site-level intervention evaluation. SZ provided project coordination and communication across New York, Kinshasa, and Lubumbashi teams, and FM provided overall country leadership including ICAP-MoH collaborations and approvals. All authors read and approved the manuscript. 


\section{Funding}

This research has been supported by PEPFAR through HRSA. Funding for this effort was provided to ICAP through HRSA grant UH6HA30739. John Ngulefac, one of the study's co-authors, is a Public Health Analyst, HRSA, and has supervised this funding.

\section{Availability of Data and Materials}

The amended rapid assessment survey used and site-level data collected for this study are available from the corresponding author upon reasonable request.

\section{Ethics Approval to Consent and to Participate}

The Columbia University Irving Medical Center's IRB reviewed this proposed research study and gave it a "non-research" determination (IRB-AAAS2598) on February 22, 2019. This determination was shared with $\mathrm{DRC} \mathrm{MoH}$, and the $\mathrm{MoH}$ also deemed this study to be of a "non-research" nature. Only site-level information was retained from interviews, and no specific individuals' names or identifying data were captured as part of any interview or discussion. Although none did, any potential interviewee could optout of the site-level interview and/or refrain from completing the amended PEPFAR assessment tool in part or in full.

\section{Consent for Publication}

The views expressed in this article are those of the authors and should not be construed to represent the positions of HRSA or of the organizations with which the authors are affiliated.

\section{Competing Interests}

The authors declare that they have no competing interests.

\section{References}

1. Wembonyama S, Mpaka S, Tshilolo L. Medicine and health in the Democratic Republic of Congo: from Independence to the Third Republic. Medecine tropicale: revue du Corps de sante colonial. 2007 Oct;67(5):447-57.

2. Mulanga C, Bazepeo SE, Mwamba JK, Butel C, Tshimpaka JW, Kashi M, Lepira F, Caraël M, Peeters M, Delaporte E. Political and socioeconomic instability: how does it affect HIV? A case study in the Democratic Republic of Congo. Aids. 2004 Mar 26;18(5):832-4. 
3. Messina JP, Emch M, Muwonga J, Mwandagalirwa K, Edidi SB, Mama N, Okenge A, Meshnick SR. Spatial and socio-behavioral patterns of HIV prevalence in the Democratic Republic of Congo. Social science \& medicine. 2010 Oct 1;71(8):1428-35.

4. PEPFAR Country Operational Plan (COP) for Democratic Republic of the Congo, Strategic Direction Summary. 2019 Apr 12

5. Ministère de la Sante République Démocratique du Congo. Ministère de la Sante. Plan Stratégique Sectoriel Sante de la Lutte Contre le VIH/SIDA 2018-2021. Avril 2018.

6. Chen L, Evans T, Anand S, Boufford JI, Brown H, Chowdhury M, Cueto M, Dare L, Dussault G, Elzinga G, Fee E. Human resources for health: overcoming the crisis. The Lancet. 2004 Nov 27;364(9449):1984-90.

7. Herbst $\mathrm{CH}$, Soucat A, Tulenko K. HIV/AIDS and human resources for health. This Changing HIV/AIDS Landscape. 2009:327.

8. Dussault G, Dubois CA. Human resources for health policies: a critical component in health policies. Human resources for health. 2003 Dec;1(1):1.

9. Chopra M, Munro S, Lavis JN, Vist G, Bennett S. Effects of policy options for human resources for health: an analysis of systematic reviews. The Lancet. 2008 Feb 23;371(9613):668-74.

10. Kavanagh MM. The politics and epidemiology of transition: PEPFAR and AIDS in South Africa. JAIDS Journal of Acquired Immune Deficiency Syndromes. 2014 Mar 1;65(3):247-50.

11. Deussom RH, Rottach E, Prabawanti C, Rahmat E, Rachmawati T, Sirajulmunir N. Health Workforce Assessment in Jakarta for Effective HIV Policy Implementation: Challenges and Opportunities toward Epidemic Control. Jurnal Ekonomi Kesehatan Indonesia. 2019 Jun 18;3(2).

12. Kaboru BB, Falkenberg T, Ndubani P, Höjer B, Vongo R, Brugha R, Faxelid E. Can biomedical and traditional health care providers work together? Zambian practitioners' experiences and attitudes towards collaboration in relation to STIs and HIV/AIDS care: a cross-sectional study. Human resources for health. 2006 Dec 1;4(1):16.

13. Brentlinger PE, Assan A, Mudender F, Ghee AE, Torres JV, Martínez PM, Bacon O, Bastos R, Manuel R, Li LR, McKinney C. Task shifting in Mozambique: cross-sectional evaluation of non-physician clinicians' performance in HIV/AIDS care. Human resources for health. 2010 Dec 1;8(1):23.

14. Marchal B, Brouwere VD, Kegels G. HIV/AIDS and the health workforce crisis: what are the next steps?. Tropical Medicine \& International Health. 2005 Apr;10(4):300-4.

15. Manzi F, Schellenberg JA, Hutton G, Wyss K, Mbuya C, Shirima K, Mshinda H, Tanner M, Schellenberg D. Human resources for health care delivery in Tanzania: a multifaceted problem. Human resources for health. 2012 Dec 1;10(1):3.

16. Ministry of Health Zambia. Ministry of Health. Zambia Population-based HIV Impact Assessment (ZAMPHIA) 2016: Final Report. February 2019.

17. Ministry of Health Malawi, Ministry of Health. Malawi Population-based HIV Impact Assessment (MPHIA) 2015-16: Final Report. October 2018. 
18. Michaels-Strasser S, Smith J, Khanyola J, Sutton R, Price T, El Sadr WM. Strengthening the Quality and Quantity of the Nursing and Midwifery Workforce: Report on Eight Years of the NEPI Project. Annals of global health. 2018;84(1):31.

19. Dräger S, Gedik G, Dal Poz MR. Health workforce issues and the Global Fund to fight AIDS, Tuberculosis and Malaria: an analytical review. Human Resources for Health. 2006 Dec 1;4(1):23.

20. Hanefeld J, Musheke M. What impact do Global Health Initiatives have on human resources for antiretroviral treatment roll-out? A qualitative policy analysis of implementation processes in Zambia. Human Resources for Health. 2009 Dec;7(1):8.

21. Dieleman M, Shaw DM, Zwanikken P. Improving the implementation of health workforce policies through governance: a review of case studies. Human resources for health. 2011 Dec;9(1):10.

22. World Health Organization. The world health report 2006: working together for health.

23. World Health Organization. Guide for rapid assessment of interactions between HIV programmes and health systems. 2014

24. PEPFAR Rapid Site-Level Health Workforce Assessment Tool, Version 1.2. PEPFAR 3.0 Human Resources for Health Technical Working Group, Washington DC. 2017. https://hrh2030program.org/pepfar_tool. Accessed 31 Mar 2020.

25. PEPFAR Monitoring, evaluation, and reporting (MER 2.0) indicator reference guide. Washington, DC. 2017. https://www.pepfar.gov/documents/organization/263233.pdf. Accessed 31 Mar 2020.

26. Gormley W, McCaffery J, Quain EE. Moving forward on human resources for health: next steps for scaling up toward universal access to HIV/AIDS prevention, treatment, and care. JAIDS Journal of Acquired Immune Deficiency Syndromes. 2011 Aug 1;57:S113-5.

27. McQuide PA, Kolehmainen-Aitken RL, Forster N. Applying the workload indicators of staffing need (WISN) method in Namibia: challenges and implications for human resources for health policy. Human resources for health. 2013 Dec 1;11(1):64.

28. Dovlo D. Using mid-level cadres as substitutes for internationally mobile health professionals in Africa. A desk review. Human resources for health. 2004 Dec;2(1):7.

29. Mathauer I, Imhoff I. Health worker motivation in Africa: the role of non-financial incentives and human resource management tools. Human resources for health. 2006 Dec;4(1):24.

30. Jones DS, Tshimanga M, Woelk G, Nsubuga P, Sunderland NL, Hader SL, St Louis ME. Increasing leadership capacity for HIV/AIDS programmes by strengthening public health epidemiology and management training in Zimbabwe. Human Resources for Health. 2009 Dec;7(1):69.

31. Kurowski C, Wyss K, Abdulla S, Yémadji N, Mills A. Human resources for health: requirements and availability in the context of scaling-up priority interventions in low-income countries. Case studies from Tanzania and Chad. 2004

32. Bajunirwe F, Twesigye L, Zhang M, Kerry VB, Bangsberg DR. Influence of the US President's Emergency Plan for AIDS Relief (PEPfAR) on career choices and emigration of health-profession graduates from a Ugandan medical school: a cross-sectional study. BMJ open. 2013 Jan $1 ; 3(5): e 002875$. 
33. Callaghan M, Ford $\mathrm{N}$, Schneider $\mathrm{H}$. A systematic review of task-shifting for HIV treatment and care in Africa. Human resources for health. 2010 Dec 1;8(1):8.

34. Lagarde M, Blaauw D. A review of the application and contribution of discrete choice experiments to inform human resources policy interventions. Human resources for health. 2009 Dec;7(1):62.

35. Porter LE, Bouey PD, Curtis S, Hochgesang M, Idele P, Jefferson B, Lemma W, Myrick R, NuwagabaBiribonwoha H, Prybylski D, Souteyrand Y. Beyond indicators: advances in global HIV monitoring and evaluation during the PEPFAR era. JAIDS Journal of Acquired Immune Deficiency Syndromes. 2012 Aug 15;60:S120-6.

36. Ndwapi N, Grignon J, Buzwani M, Ledikwe J, Malefho K, Mbayi B, Mogasha C, Nyangah R, Smith M, Semo B. Improving efficiency in project implementation through a PEFFAR-funded initiative to strengthen governance of Ministry of Health programs. InProceedings of the 20th International AIDS Conference 2012.

37. Wyss K. An approach to classifying human resources constraints to attaining health-related Millennium Development Goals. Human resources for health. 2004 Dec;2(1):11.

38. Anand S, Bärnighausen T. Human resources and health outcomes: cross-country econometric study. The Lancet. 2004 Oct 30;364(9445):1603-9.

39. Sturke R, Harmston C, Simonds RJ, Mofenson LM, Siberry GK, Watts DH, Mclntyre J, Anand N, Guay L, Castor D, Brouwers P. A multi-disciplinary approach to implementation science: the NIH-PEPFAR PMTCT implementation science alliance. JAIDS Journal of Acquired Immune Deficiency Syndromes. 2014 Nov 1;67:S163-7.

40. Kavanagh MM, Dubula-Majola V. Policy change and micro-politics in global health aid: HIV in South Africa. Health policy and planning. 2019 Feb 1;34(1):1-1.

41. Biradavolu M, Deshpande A, Guida M, Kearns M, Trasi R, Wilhelmsen S. Fostering PEPFAR Sustainability through Leadership, Management, and Governance. 2017.

\section{Supplementary Files}

This is a list of supplementary files associated with this preprint. Click to download.

- Exhibits.docx 